\title{
The Off-Label Use of Hydroxychloroquine in Prophylaxis and Treatment of COVID-19
}

\section{ASHISH PAUL}

Coronavirus pandemic has covered the entire globe only in a few months and has been declared as a public health emergency due to a very high morbidity as well as mortality rate. Till date no curative measure is available for this fatal disease and only social distancing has been of some help in prevention. However, anti-malarial drug hydroxychloroquine is being tried for prophylaxis and treatment at early stages of the disease although it is not an approved indication for this drug which has given some hope to health care professionals and the affected population.

KEYWORDS: Coronavirus, COVID-19, Hydroxychloroquine

\section{INTRODUCTION}

Coronavirus disease 2019 (COVID-19) outbreak caused by the novel corona virus was declared as a Public Health Emergency of International Concern on 30 January 2020 and recognized as a pandemic by the World Health Organization on March 11, 2020. As per the coronavirus disease 2019 (COVID-19) Situation Report -88 by WHO dated April 17, 2020, the number of reported confirmed cases exceeded 2 million globally. Subjects infected with COVID-19 often present with dry cough, fever, and fatigue at onset, myalgia, sore throat, nasal congestion, runny nose, headache and diarrhoea in mild to moderate cases and may have a rapid progression to acute respiratory distress syndrome, septic shock, uncorrectable metabolic acidosis, coagulopathy, and multiple organ failure in severe cases. ${ }^{2,3}$ No available drug or vaccine is specifically approved for this virus in clinical practice, so it is an important task to find and develop such drugs that can inhibit SARS-CoV-2 infection. However, before a drug or vaccine is available in the market, it must go through multiple phases of drug development including safety and efficacy trials and this cycle is a time intensive procedure, so the selection of drugs with anti-SARS-CoV-2 activity among existing clinically available drugs has become a faster option at present. Till now, many clinical trials involving new coronavirus therapy have been registered nationwide, mainly involving antimalarials and antiviral drugs.

Hydroxychloroquine was synthesized in 1946 and marketed in 1955 as a safe alternative to chloroquine. ${ }^{4}$
It is currently mainly used for rheumatoid arthritis, juvenile chronic arthritis, mild systemic and discoid lupus erythematosus and the suppression and treatment of malaria, and photosensitivity disorders. The pharmacokinetics and clinical adverse effects of hydroxychloroquine have been established; however, since the target population of new indications, dosage and time are different from the previous application, there is a risk of adverse reactions and even serious adverse consequences. Therefore, it is necessary to comprehensively review the pharmacological effects, metabolism, and distribution in vivo, poisoning and toxicological mechanism of chloroquine drugs, enhance the understanding of medical workers, forensic doctors and the public on acute toxicity of this drug, timely detect and reasonably treat serious adverse reactions, reduce adverse reactions as well as even death caused by drug use.

Many trials assessing the efficacy of hydroxychloroquine against COVID-19 have been registered worldwide. Chen $\mathrm{Z}$ et al conducted a placebo controlled randomized trial with two different doses of hydroxychloroquine in 62 subjects who reported small improvements in body temperature and cough in the treatment group taking the higher dose. ${ }^{5}$

Off-label use of a drug refers to a situation where it is prescribed for an indication, route of administration or patient group not included in the labelling document of the drug. Till now, no medication has been approved by 
Therapeutic Goods Administration for COVID-19. ${ }^{6}$

Due to weak evidence of clinical benefits of hydroxychloroquine against COVID-19 and poor clarity on antiviral mechanism of action of this drug, the drug manufacturers have not included this disease as an indication in the product document. It is proposed that hydroxychloroquine could inhibit viral entry into host cells and interferes with the acidification of host cell lysosomes. Yao X et al. ${ }^{7}$ and Liu Y et al. ${ }^{8}$ conducted invitro studies and reported that hydroxychloroquine can inhibit the replication of SARS-CoV-2.

Although, long term use of hydroxychloroquine can result in serious cutaneous adverse reactions, fulminant hepatic failure, maculopathies, macular degeneration, retinopathy, torsade de pointes, ventricular tachycardia, cardiomyopathy and cardiac failure but it has been permitted by the USFDA and also advocated by the Indian Council for Medical Research because no definite treatment for COVID-19 is available yet.9,10 Since the start of this novel coronavirus outbreak, hydroxychloroquine has been applied in clinical practice and is expected to play an important role in controlling the pandemic. Researchers are involved in conducting trials of hydroxychloroquine but there is scarcity of evidence to support its efficacy in preventing COVID-19. Prophylaxis with hydroxychloroquine against COVID-19 needs to be thoroughly evaluated in observational studies and high quality randomized controlled trials. It is also necessary to strengthen the understanding of this drug and their toxicological characteristics.

\section{CONCLUSION}

There has been an urgent worldwide demand for treatments as a result of the essentially untreatable coronavirus disease, but no intervention can be assumed to be efficacious. The use of hydroxychloroquine is being trialled to investigate its capability in inhibiting coronavirus. However, it is also important to consider the toxicological profile before rational use of hydroxychloroquine. A breakthrough in the effective treatment with vaccine or drug for coronavirus infection may take time, but prevention with empiric drugs that target specific structures in the virus along with supportive measures can be of great help and relief. Along with efficacy studies, it is also important to check the safety profile of drugs like hydroxychloroquine. There is a need of conducting invitro studies as well as in-vivo randomized controlled trials of hydroxychloroquine to find out how effective it can be in preventing and treating coronavirus infections at doses which do not cause any potential adverse reactions in humans.

\section{REFERENCES}

1. WHO. (Online PDF). Available from: https://www.who.int/docs/defaultsource/coronaviruse/situation-reports/20200417sitrep-88-covid191b6cccd94f8b4f219377bff55719a6ed.pdf?sfursn=ebe7 8315 6. [Last Accessed on $15^{\text {th }}$ March, 2020]

2. Huang C, Wang Y, Li X, Ren L, Zhao J, Hu Y, et al. Clinical features of patients infected with 2019 novel coronavirus in Wuhan, China. Lancet. $2020 \mathrm{Feb}$ 15;395(10223):497-506. doi: 10.1016/So1406736(20)30183-5.

3. Zhang JJ, Dong X, Cao YY, Yuan YD, Yang YB et al. Clinical characteristics of 140 patients infected with SARS-CoV-2 in Wuhan, China. Allergy. 2020; 00:1-12. https://doi.org/10.1111/all.1423.

4. Tzekov, R. Ocular toxicity due to chloroquine and hydroxychloroquine: Electrophysiological and visual function correlates. Doc Ophthalmol. 2005;110:111-12.

5. Chen Z, Hu J, Zhang Z, et al. Efficacy of hydroxychloroquine in patients with COVID-19: results of a randomized clinical trial. Version 2. med Rxiv 2020.03.22.20040758. [Preprint.] 10.1101/2020.03.22.20040758.

6. Seale JP. Off-label prescribing. Med J Aust 2014;200(2). doi: 10.5694/mja13.00184.

7. Lenzer J. Covid-19: US gives emergency approval to hydroxychloroquine despite lack of evidence. BMJ 2020;369:m1335. 10.1136/bmj.m1335 32238355.

8. Indian Council for Medical Research. Recommendation for empiric use of hydroxychloroquine for prophylaxis of SARS-CoV-2 infection. (Online PDF). Available from: https://icmr.nic.in/sites/default/files/upload docume nts/HCQ Recommendation 22March final MM V2. pdf. [Last Accessed on $15^{\text {th }}$ March, 2020]

9. Yao X, Ye F, Zhang M, et al. In vitro antiviral activity and projection of optimized dosing design of hydroxychloroquine for the treatment of Severe Acute Respiratory Syndrome Coronavirus 2 (SARSCoV-2). Clin Infect Dis 2020;ciaa237. doi: 10.1093/cid/ciaa237.

10. Liu J, Cao R, Xu M, et al. Hydroxychloroquine, a less toxic derivative of chloroquine, is effective in inhibiting SARS-CoV-2 infection in vitro. Cell Discov 2020;6:16. doi: 10.1038/s41421-020-0156-o. 
Source of support: Nil, Conflict of interest: None declared

Cite this article as:

Paul A. The Off-Label Use of Hydroxychloroquine in Prophylaxis and

Treatment of COVID-19. Int Healthc Res J. 2020;4(1):7-9. https://doi.org/10.26440/IHRJ/0401.04339

AUTHOR AFFILIATIONS: ( ${ }^{*}$ Corresponding Author)

1. MBBS, General and Consultant Physician, Ludhiana, India

Corresponding Address:

H. No. 21, Block C

Contact corresponding author at: ashishpaul315[at]gmail[dot]com

GK Estate

Tibba Road

Ludhiana 